NOVEMBER 2021

CEDIL Methods Working Paper 5

\title{
The strategic use of evidence and gap maps to build evidence architecture
}


CEDIL methods working paper: The strategic use of evidence and gap maps to build evidence architecture

\section{About CEDIL}

The Centre of Excellence for Development Impact and Learning (CEDIL) is an academic consortium supported by the UK government through UK Aid. The mission of the Centre is to test innovative methodologies in evaluation and evidence synthesis and promote evidence-informed development. CEDIL-supported projects fall into three programmes of work: evaluating complex interventions, enhancing evidence transferability, and increasing evidence use.

\section{CEDIL methods working papers}

The CEDIL methods working paper series offers innovative research methods for developing impact evaluation and evidence synthesis work in low- and middle-income countries.

\section{About this working paper}

Please direct any comments or queries to the corresponding author, Howard White, at hwhite@campbellcollaboration.org

Suggested citation: White, H. (2021) The Strategic Use of Evidence and Gap Maps to Build the Evidence Architecture. CEDIL Methods Working Paper 5. London and Oxford: Centre of Excellence for Development Impact and Learning (CEDIL). Available at: https://doi.org/10.51744/CMWP5

This project was funded by the Centre of Excellence for Development Impact and Learning (CEDIL), supported by UK aid from the UK Government. The views expressed in this working paper do not necessarily reflect the UK Government's official policies.

Cover design: PhilDoesDesign

Copyright: (C) 2021 
CEDIL methods working paper: The strategic use of evidence and gap maps to build evidence architecture

\section{CEDIL methods working paper: The strategic use of evidence and gap maps to build evidence architecture}

\section{Author}

Howard White

CEO, Campbell Collaborations, and Research Director, CEDIL 
CEDIL methods working paper: The strategic use of evidence and gap maps to build evidence architecture

\section{Contents}

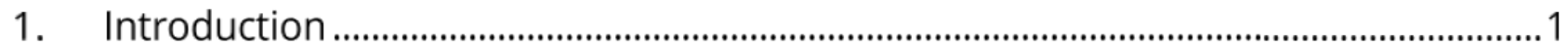

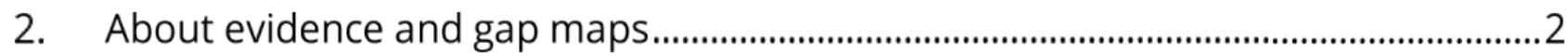

3. Overview of the use of evidence and gap maps ............................................................ 4

4. An approach to using evidence and gap maps to build evidence architecture .......7

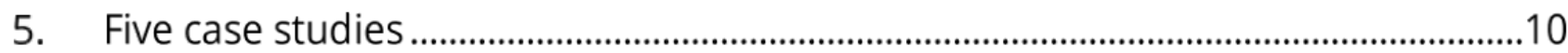

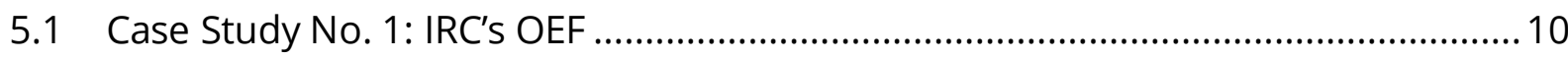

5.2 Case Study No. 2: Evidence for disability in development ........................................... 11

5.3 Case Studies Nos. 3 and 4: The UK What Works Centres - CHI and YEF...................14

5.4 Case Study No. 5: Child protection in institutional settings ........................................ 15

6. Lessons about the use of evidence and gap maps...................................................18

\section{List of tables and figures}

Table 1: Uses of different evidence and gap maps 6

Figure 1: Snapshot of a section of a map relating to evidence on interventions in the area of violence against children. 3

Figure 2: The knowledge brokering pyramid ........................................................................ 8

Figure 3: The Education Endowment Foundation Teacher and Learning Toolkit.......................9

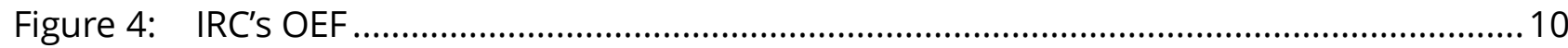

Figure 5: Disability evidence portal ................................................................................... 12

Figure 6: Including cell-wise evidence summaries in an evidence and gap map......................16 
CEDIL methods working paper: The strategic use of evidence and gap maps to build evidence architecture

\section{Acronyms and abbreviations}

CEDIL Centre of Excellence for Development Impact and Learning

$\mathrm{CHI} \quad$ Centre for Homelessness Impact

DFID UK Department for International Development

FCDO UK Foreign, Commonwealth and Development Office

ICED International Centre of Evidence for Disability

IRC International Rescue Committee

NICE National institute for Health and Care Excellence

OEF $\quad$ Outcomes to Evidence Framework

PICOS Population, intervention, comparisons, outcomes and study

REA Rapid evidence assessment

UNICEF United Nations Children's Fund

WHO World Health Organization

YEF Youth Endowment Foundation 
CEDIL methods working paper: The strategic use of evidence and gap maps to build evidence architecture

\section{Introduction}

The demand for evidence has been growing for some time. Evidence mapping has emerged as an important part of the response to this demand. A number of organisations have adopted various approaches to evidence mapping, including the EPPI Centre, the US Department of Veterans' Affairs, the Collaboration for Environmental Evidence (James et al., 2016), and the International Initiative for Impact Evaluation (3ie) (Snilstveit et al., 2013); see Saran and White (2018) for a review of these approaches.

Such evidence and gap maps have emerged as an important way to make evidence available to decision-makers. However, while evidence and gap maps have benefits over other, less systematic or accessible, approaches to reporting evidence availability, they have the drawback that they take the user to research papers. Many decision-makers, and sometimes also researchers, do not have the time, or possibly the skills, to read or to make use of research papers, especially if they are presented with say 10-12 papers on the same topic. Hence, evidence and gap maps may be better used as a tool or stepping-stone for producing evidence-based decision-making products that do not require the decision-maker to directly read and interpret the research evidence. This paper describes this approach.

The paper is intended for both researchers and research commissioners. Research commissioners are encouraged to commission evidence and gap maps as a basis for making more strategic research investments. Researchers who intend for their research to inform policy and practice should seek collaboration with 'user commissioners'. User commissioners are research commissioners who commission research products with an intended policy use in mind. Examples of these user commissioners are given in the case studies in this paper.

Sections 2 and 3 of the paper provide a short introduction to evidence and gap maps and Section 4 explains how they can be used strategically to build evidence architecture. Section 5 presents fives case studies that show this approach in action. Section 6 concludes with some general lessons. 
CEDIL methods working paper: The strategic use of evidence and gap maps to build evidence architecture

\section{About evidence and gap maps}

Mapping is an evidence synthesis approach that aims to describe what research evidence is available that is relevant to a particular research or policy question. Evidence maps follow the same systematic principles as a systematic review. That is: (1) a clear statement of the scope of the map, based on the intended population, intervention, comparisons, outcomes and study designs (PICOS), which may be adapted depending on the nature of the studies or other products to be included; (2) a comprehensive and systematic search; (3) double screening against explicit inclusion and exclusion criteria; (4) double coding of all included studies; and (5) systematic analysis and reporting of all coded data. A map sets out the number of studies, and their distribution according to various characteristics, such as study design, geography and sub-populations.

Pictorial representations of the evidence identified by evidence mapping started to appear about 10 years ago. An early example is the evidence-based policing matrix (Lum et al., 2011). Maps in the form produced by 3ie have now become the most common approach (see Snilstveit et al. (2013) for a discussion). While mapping is an approach which may be applied to any research question (e.g. prevalence, risk and protective factors, and the consequences of exposure to an adverse event), the 3ie maps - labelled evidence gap maps - have been maps of effectiveness studies (here referred to as 'effectiveness maps').

The map is a matrix or a table in which the row headings are intervention names (sometimes organised into categories and sub-categories) and the column headings are outcomes (again sometimes organised into categories and sub-categories). Each cell in the matrix contains bubbles to represent studies. In 3ie maps there are typically separate bubbles for primary studies and reviews, with critical appraisal provided for the reviews. Crucially, the maps are interactive. The user can click on a cell to obtain a list of studies in that cell and to access the source for each study (the pdf, or the journal or working paper page).

The 3ie maps studies from the field of international development. In 2018, the Campbell Collaboration began to explore including evidence and gap maps among its products, developing an approach which is similar to that of 3ie, though Campbell uses different software and has produced a broader range of types of map, for example including process evaluations as well as impact evaluations. The EPPI Centre was commissioned to produce software to produce maps, EPPI Mapper, which is linked to their review software, EPPI Reviewer. A variety of maps are now registered with Campbell and guidance for producing evidence and gap maps is available (White et al., 2020b).

An example of a Campbell map is shown in Figure 1: this gives a snapshot of a section of a map of effectiveness studies on the subject of tackling violence against children (Pundir et al., 2020 ). Each cell in the map shows the number of studies - the more studies the bigger the bubble. The studies are categorised in a cell by what is called the 'segmenting variable'. In this case the map distinguishes between primary studies and reviews, which are further divided by the confidence in the study findings, based on a critical appraisal of the included studies. The map is interactive: the user can click on a cell to obtain a list of the studies in that cell. Clicking on any study in the list gives the details of the study and a link to the source, i.e. the underlying study. The studies in the map are also searchable by using a search function. 
CEDIL methods working paper: The strategic use of evidence and gap maps to build evidence architecture

\section{Figure 1: Snapshot of a section of a map relating to evidence on interventions in the area of violence against children}

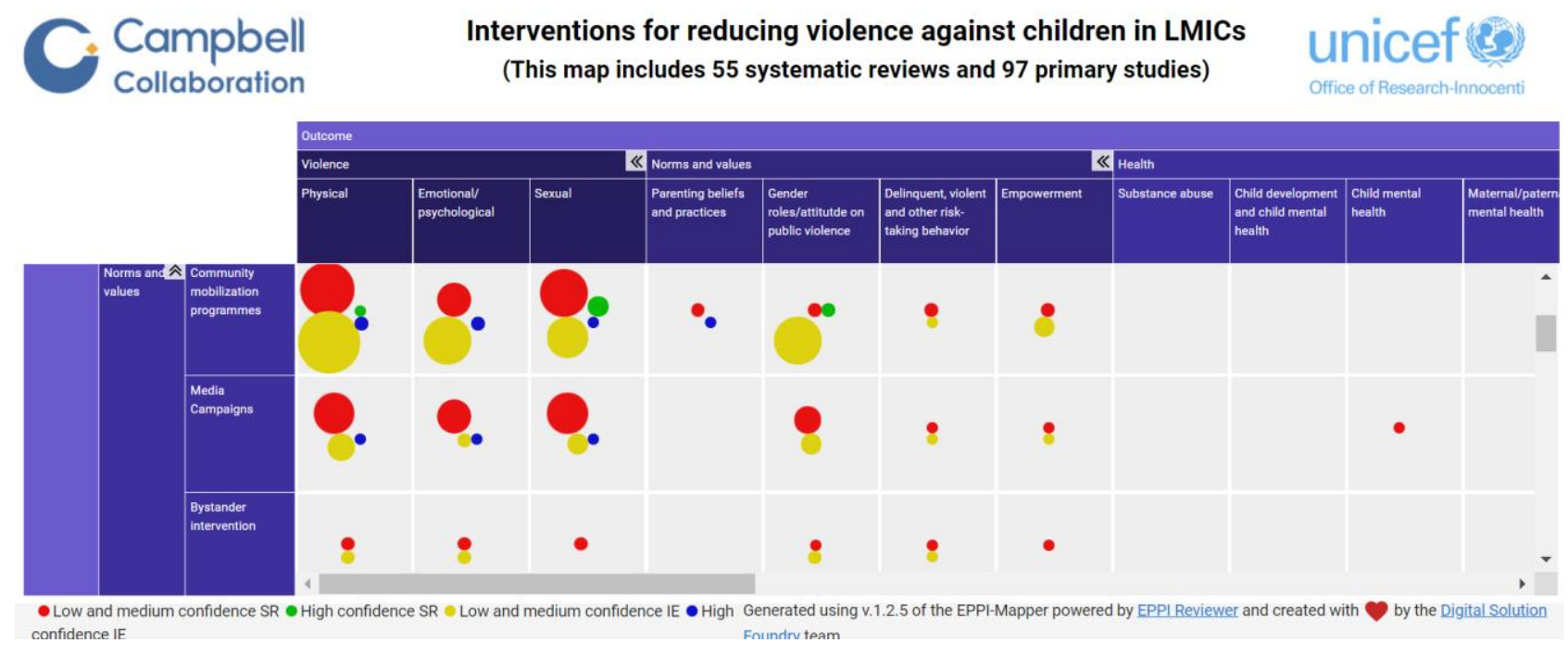

Source: Pundir et al., (2020)

Link to map: https://onlinelibrary.wiley.com/pb-

assets/assets/18911803/Campbell_Interventions-for-reducing-violence-against-children.HTML

This example is an effectiveness map of primary studies and reviews. However, a range of other types of map are produced, including:

- megamaps, which have a very broad scope and which contain only reviews and other maps (such as a megamap of child welfare in developing countries, Saran et al. (2020a));

- maps which include process evaluations (e.g. White et al. (2018) for homelessness);

- maps of tools, methods and metrics for agriculture-nutrition linkages (e.g. Sparling et al. (2019) and Sparling et al. (2021)); and

- country evaluation maps of all evaluations in a particular country (e.g. White at al. (2021 b) for Uganda).

The advantage of these evidence and gap maps is that they make the research evidence discoverable and accessible. Since maps are based on a comprehensive and systematic search, following usual systematic review principles, they can uncover much more evidence than commissioners and users are usually aware of. And, as the maps are interactive, providing the user access to the underlying research, they increase access to - and so use of the evidence.

As described in the case studies below, the maps themselves can be used directly by decisionmakers to navigate and use the evidence. However, an emerging use of the maps is the more strategic role of building evidence architecture. 


\section{Overview of the use of evidence and gap maps}

Evidence and gap maps can be used in a variety of ways:

- They can be made available to decision-makers to facilitate their access to evidence. This has been the approach for many of the maps commissioned through 3ie.

- They can be used to identify areas for which more detailed mapping can usefully be commissioned.

- They can be used to identify cells or other clusters with primary studies but no recent review, so new or updated systematic reviews can be commissioned.

- If reviews are commissioned then the studies in map used for the included studies in the review, so a sequenced approach of mapping follow by review removes the need for a separate search.

- The evidence in maps may be used to inform programme design or funding decisions for programmes or research.

- They can be used to identify studies to provide content for evidence-based decisionmaking products.

Examples of these uses include the following cases. The first five of these examples are elaborated in the case studies below, and all of them are summarised in Table 1:

- The International Rescue Committee (IRC) developed a series of evidence and gap maps to provide content for the Outcomes to Evidence Framework (OEF), which is intended to enable IRC programme managers to make evidence-informed decisions.

- The Centre of Excellence for Development Impact and Learning (CEDIL) commissioned a map of maps, which identified disability as an area without an evidence map; such a map was then commissioned. The resulting map was the basis for a series of rapid reviews, which were then used as one input to construct an evidence portal.

- Both the Centre for Homelessness Impact $(\mathrm{CHI})$ and the Youth Endowment Foundation (YEF) commissioned evidence maps as a means to identify studies to build their evidence portals.

- Giving Evidence/Porticus produced evidence summaries as a basis for an evidence manual to accompany the map they commissioned on child protection.

- A megamap - that is, a map of systematic reviews only - of child welfare in low- and middle-income countries identified a lack of reviews on violence against children (Saran et al., 2020a). The violence against children evidence and gap map (Figure 1) was commissioned to take a closer look at what evidence is available on that topic (Pundir et al., 2020). The United Nations Children's Fund (UNICEF) Innocenti Research Centre published briefs on the main intervention areas covered by both the megamap and the violence against children map to encourage use by UNICEF staff. ${ }^{1}$

1 The briefs can all be found at https://www.unicef-irc.org/publications/series/research-briefs/. 
CEDIL methods working paper: The strategic use of evidence and gap maps to build evidence architecture

- IMMANA is a research programme that focuses on tools, methods and metrics for assessing linkages between agriculture and nutrition. A map of existing studies was used to inform funding for new studies (Sparling et al., 2021). In the application for funding, applicants were required to justify their proposed study with reference to the map.

- Mastercard Foundation commissioned a map of youth employment which will be used as one input to help inform programme funding and design decisions.

As Table 1 clearly shows, evidence and gap maps are not an end in themselves, nor are they produced solely for research purposes. Maps are used for a range of purposes, from commissioning new studies to directly informing programming decisions, to being a basis for the production of evidence-based decision-making products. 
CEDIL methods working paper: The strategic use of evidence and gap maps to build evidence architecture

Table 1: Uses of different evidence and gap maps

\begin{tabular}{|c|c|c|c|c|c|c|c|c|c|}
\hline & $\begin{array}{l}\text { Campbell- } \\
\text { UNICEF } \\
\text { child } \\
\text { welfare } \\
\text { megamap }\end{array}$ & $\begin{array}{l}\text { CEDIL-3ie } \\
\text { map of } \\
\text { maps }\end{array}$ & $\begin{array}{l}\text { CEDIL } \\
\text { disability } \\
\text { map }\end{array}$ & $\mathrm{CHI}$ & $\begin{array}{l}\text { Giving } \\
\text { Evidence } \\
\text { and } \\
\text { Porticus }\end{array}$ & IMMANA & $\begin{array}{c}\text { IRC } \\
\text { outcomes to } \\
\text { evidence } \\
\text { framework }\end{array}$ & $\begin{array}{l}\text { Mastercard } \\
\text { Foundation }\end{array}$ & YEF \\
\hline $\begin{array}{l}\text { Identify more detailed } \\
\text { mapping to be } \\
\text { commissioned }\end{array}$ & $x$ & $x$ & & & & & & & \\
\hline $\begin{array}{l}\text { Studies in map used } \\
\text { for reviews }\end{array}$ & $x$ & & $x$ & $x$ & & & & & $x$ \\
\hline $\begin{array}{l}\text { Inform programme } \\
\text { design }\end{array}$ & & & & & & & $x$ & $x$ & \\
\hline $\begin{array}{l}\text { Inform primary study } \\
\text { funding decisions }\end{array}$ & & & $x$ & & $x$ & $x$ & & & $x$ \\
\hline $\begin{array}{l}\text { Inform primary study } \\
\text { design decisions }\end{array}$ & & & $x$ & & & & & & \\
\hline $\begin{array}{l}\text { Provide content for } \\
\text { evidence-based } \\
\text { decision-making } \\
\text { products }\end{array}$ & & & $x$ & $x$ & $x$ & & $x$ & & $x$ \\
\hline
\end{tabular}




\section{An approach to using evidence and gap maps to build evidence architecture}

The number of papers published in peer reviewed journals grew by nearly 2.6 million in $2018 .^{2}$ The rapid growth in research in general, and evidence on 'what works' in particular (White, 2019), means that it is very difficult for both researchers and decision-makers to keep abreast of growing bodies of literature. In addition, there are well-known barriers o decisionmakers using research literature, on account of the lack of discoverability and accessibility of much of this research. Lack of accessibility means both that papers may be behind paywalls, and that papers may be written in a technical style that lay readers cannot readily understand. All of these factors underline the desirability of knowledge translation and knowledge brokering, to help facilitate the use of evidence.

Knowledge brokering can take two forms. The first is knowledge brokering services, where knowledge brokers respond to specific requests for evidence. Having up-to-date evidence and gap maps greatly facilitates the work of such knowledge brokers. The second form of knowledge brokering is the institutionalisation of the use of evidence through the production of evidence-based decision-making products. Evidence and gap maps can play an important role in the development of such products.

Evidence-based decision-making products sit at the top of the knowledge brokering pyramid (Figure 2). I distinguish three types of evidence-based decision-making products: evidence portals, guidance and checklists. Evidence portals, also called toolkits or clearinghouses, are interactive platforms which present findings on the effectiveness of different interventions in a given sector or area, such as education or homelessness. Guidance or guidelines provide evidence-based recommendations on policy and practice. Checklists indicate a list of practices or procedures to be followed. The degree of agency of the decision-maker differs across these three types. Portals present evidence that enables the decision-maker to decide what to do. In the case of guidelines, experts have reviewed the evidence and made recommendations. Checklists are a 'do it this way' approach. Other differences include the fact that evidence portals are more oriented towards what works, whereas guidance and checklists are more oriented towards how to do it, though that distinction is not clear cut.

${ }^{2}$ https://ncses.nsf.gov/pubs/nsb20206/publication-output-by-field-of-science 
CEDIL methods working paper: The strategic use of evidence and gap maps to build evidence architecture

\section{Figure 2: The knowledge brokering pyramid}

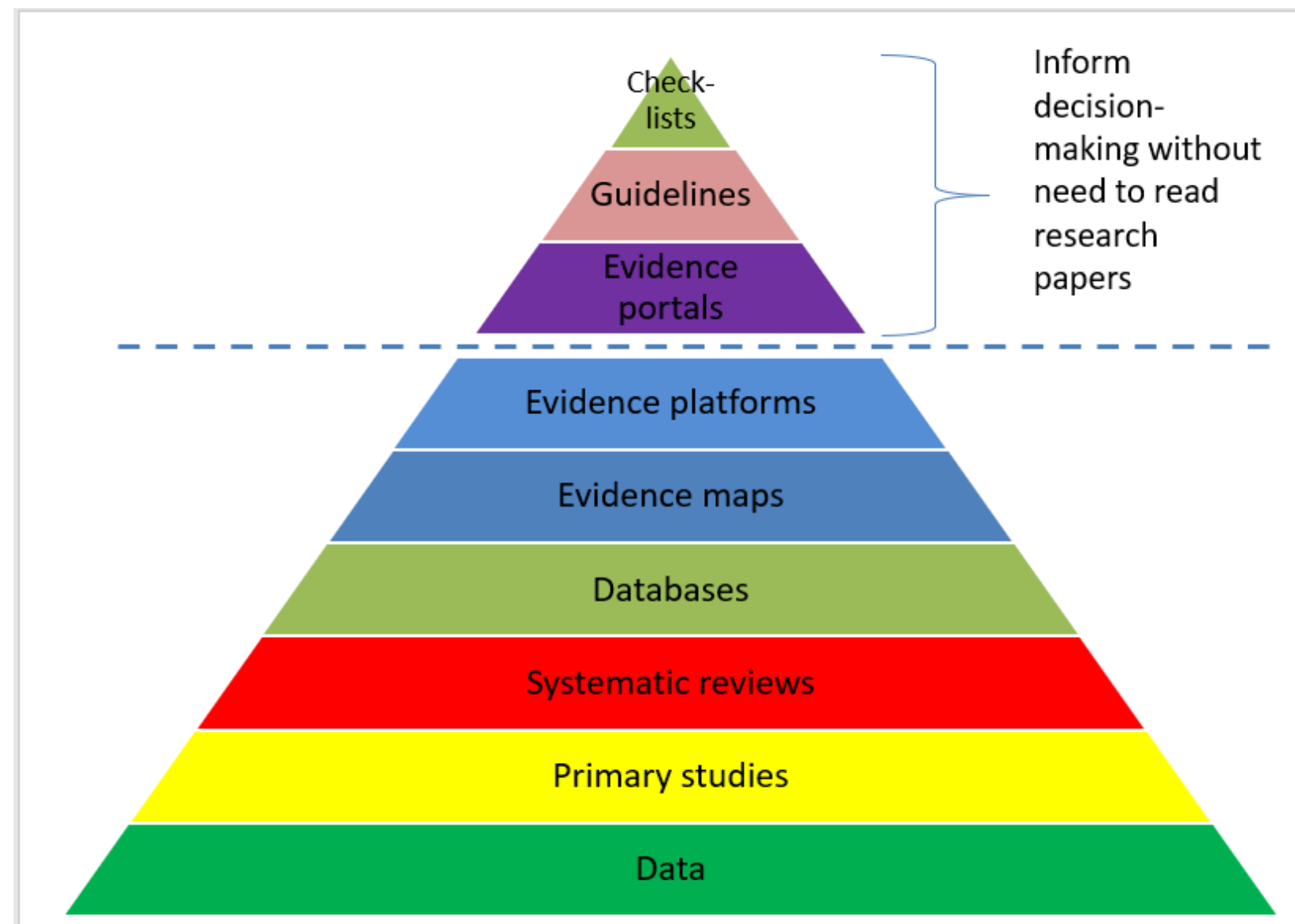

Source: White (2020a)

The strongest emerging example of an evidence portal is the UK What Works Centres.

Following the lead of the Education Endowment Foundation's Teaching and Learning Toolkit, ${ }^{3}$ these evidence portals (called toolkits or intervention tools) have three levels: (i) a top level showing the impact of different approaches (Figure 3); (ii) a second level that provides more information on the approach, issues to consider for implementation and so on; and (iii) a third level that is the underlying technical report. The technical report, which is used as the basis for the content of the top two levels of the portal, is based on systematic reviews.

The use of guidance is best established in the health sector. At the international level the World Health Organization (WHO) publishes guidelines which often influence national guidelines. Some countries have their own guideline process, such as England's National Institute for Health and Care Excellence (NICE). Guidelines are increasingly being developed for other sectors, notably by What Works Centres. Checklists are also common in health, but, as documented by Gawande (2009), they can be put to good use in a wide range of sectors.

\footnotetext{
${ }^{3}$ The first example of such an approach is the United States' What Works Clearinghouse for education: https://ies.ed.gov/ncee/wwc/. This differs in some respects from the Education Endowment Foundation's approach; see Gough and White (2018) for a discussion of the approaches of different what works centres.
} 
CEDIL methods working paper: The strategic use of evidence and gap maps to build evidence architecture

Figure 3: The Education Endowment Foundation Teacher and Learning Toolkit

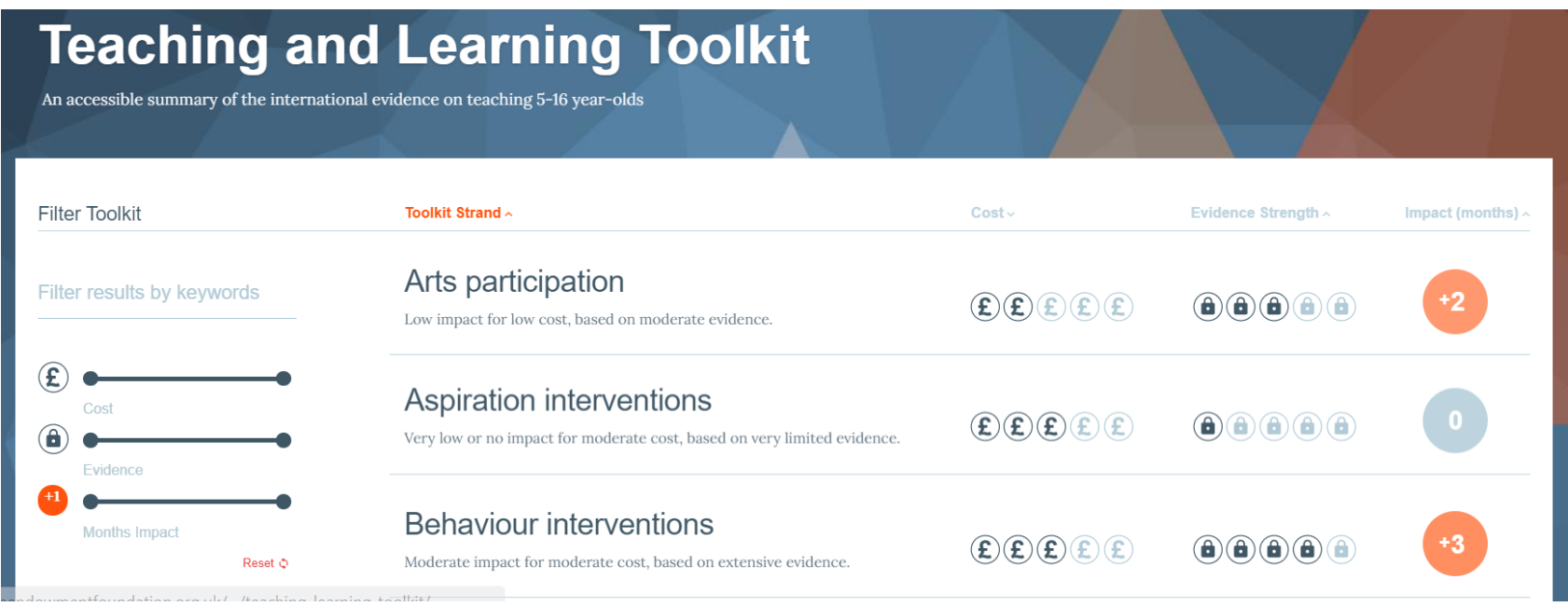

Source: https://educationendowmentfoundation.org.uk/evidence-summaries/teachinglearning-toolkit/

These evidence-based decision-making products sit at the top of the knowledge brokering pyramid. But the top of the pyramid can only be as strong as its base. Evidence-based decision-making products should be based on systematic reviews. For example, as described in the WHO Handbook on Guideline Development, the guideline process begins with a search for relevant systematic reviews; if none are available, a review is commissioned (WHO, 2010).

At the base of the pyramid are data, which get analysed in studies, which are in turn summarised in reviews. These studies and reviews are curated in databases; or they are more heavily created so that they can be portrayed in maps or in collections on evidence platforms. A critical break appears in regard to the top three levels of the pyramid, which are evidencebased decision-making products. The crucial difference between the top three levels and the levels below them is that these products allow the decision-maker to make evidence-based decisions without having to read the underlying research papers. In contrast, databases, maps and evidence platforms take the user back to the research.

A new What Works Centre starting out the process of developing an evidence portal is well advised to start by producing an evidence and gap map. The evidence and gap map surveys the state of the base of the pyramid. The map identifies: (i) existing reviews which can be used to produce technical reports for the portal; (ii) topics with many studies but no recent review, so a review can be commissioned which reviews the studies in the map for that topic; and (iii) topics for which there are few or no primary studies, which will thus need to be commissioned before it is worth commissioning a review. 
CEDIL methods working paper: The strategic use of evidence and gap maps to build evidence architecture

\section{Five case studies}

These case studies document instances of how evidence and gap maps have been used as the basis for developing top-of-the-pyramid evidence-based decision-making products.

\subsection{Case Study No. 1: IRC's OEF}

IRC'S OEFis an online interactive tool that helps programme managers to find evidence to inform programme design - that is, it is an example of an evidence portal. As shown in Figure 4, the user picks a domain (education in this example); the tool then highlights the causal chain for that domain. This causal chain includes a number of outcomes. Here the outcome ' 6 to 14 year olds have literacy, numeracy, and social-emotional skills, according to their development potential' is highlighted.

In Figure 4 the yellow cell is the one which has been clicked on, with the black cells picking out the associated causal chain. The left-hand panel shows the name of the selected box (which here is ' 6 to 14 year olds have literacy, numeracy, and social-emotional skills, according to their development potential'), and a list of the type and number of documents available, with a pull-down menu to access content.

\section{Figure 4: IRC's OEF}

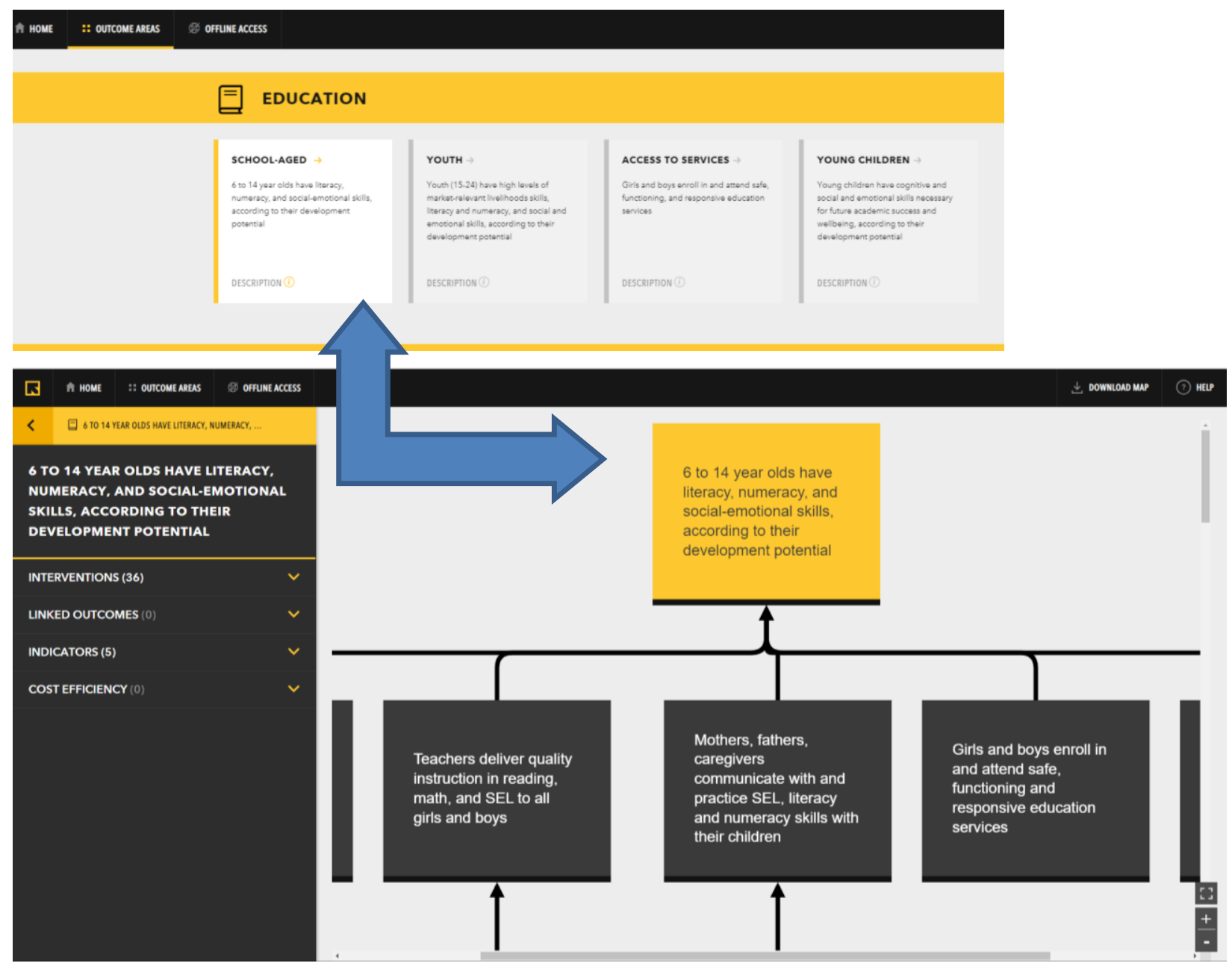

Source: http://oef.rescue.org/\#/outcome/4? k=gjhi2o 
CEDIL methods working paper: The strategic use of evidence and gap maps to build evidence architecture

The OEF hosts comprehensive theories of change aligned with the five IRC domains and a cross-cutting domain of service delivery. There is a clear logic to the OEF: users are first prompted to think about the key outcomes associated with a domain, followed by the logical sub-outcome causal pathways, and then they are prompted to consider the evidence around potential interventions that could be implemented to achieve these outcomes. Users access the evidence, sourced from the maps, by clicking on any box in the pathway, which then opens up an evidence card on the right-hand panel. The panel highlights key interventions, the strength of evidence for the interventions, the corresponding literature, linked outcomes and indicators, cost efficiency studies (though frequently there are few or no such studies), and/or resources like the IRC core indicator guidance notes.

The OEF is based on an evidence mapping. In consultation with 3ie, IRC produced a series of evidence maps to identity research evidence associated with five key domains (health, education, economic wellbeing, safety, and power), as well as three cross-cutting maps on cash transfer interventions, service delivery interventions, and interventions that are directly relevant to humanitarian emergencies.

These maps were used by IRC for a variety of purposes, such as writing proposals, developing programme guidance, and keeping up to date with the latest research, as well as informing strategic planning. One key role of the maps was to support the development of the OEF. IRC has recently been updating the maps in order to undertake a refresh of the OEF.

\subsection{Case Study No. 2: Evidence for disability in development}

This case study presents a long chain of evidence products supporting subsequent products. It also shows how CEDIL, as a flexible and responsive funding window, can support a series of work which builds on earlier work, especially if research teams are willing to negotiate different funding sources. In this case, a map of maps led to the production of three evidence and gap maps. One of these maps, on disability, was used as the basis for three systematic reviews, which are now being used to inform the content of an evidence portal (Figure 5). 
CEDIL methods working paper: The strategic use of evidence and gap maps to build evidence architecture

\section{Figure 5: Disability evidence portal}

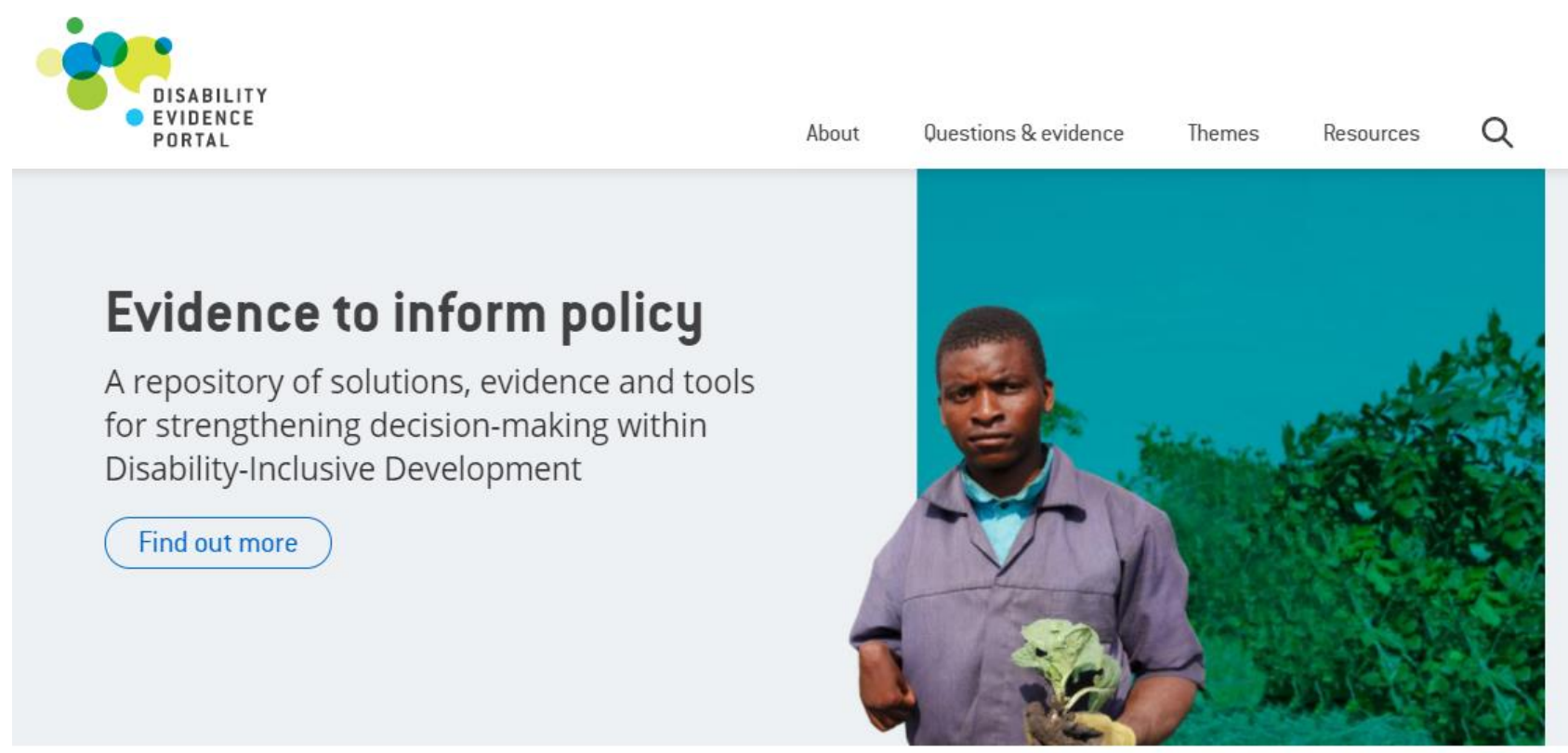

Core themes
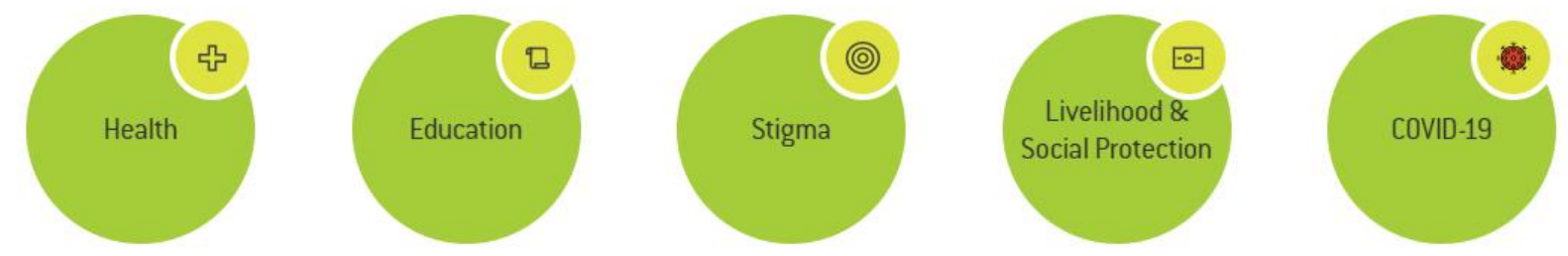

Source: https://www.disabilityevidence.org/

The case study begins with the 3ie-CEDIL map of maps. CEDIL is a UK aid-funded research programme that aims to fill several gaps:

- methods gaps - innovative methods to address evaluation questions that are not adequately addressed by current methods for impact evaluation and evidence synthesis;

- evidence gaps - sectors for which there is relatively little evidence; and

- knowledge translation gaps - innovative methods for translating knowledge for use by decision-makers and practitioners.

To address the second of these gaps - evidence gaps - CEDIL is producing a series of evidence and gap maps. To identify the topic areas for these evidence and gap maps, CEDIL produced a map of maps $-{ }^{4}$ that is, a map of all evidence and gap maps in the field of international development. The map of maps identified 73 published and ongoing maps, which were concentrated in certain sectors (Phillips et al., 2017). Specifically, health, nutrition and population was the sector with the highest number of evidence maps, followed by agriculture and rural development, education, and climate change and the environment. There was no map at all for transportation, and only one map for energy, as well as for economic policy.

${ }^{4}$ The map was produced by 3ie, which is a member of the CEDIL consortium. 
CEDIL methods working paper: The strategic use of evidence and gap maps to build evidence architecture

There was also a relatively small number of maps for information and communications technology, humanitarian programmes, and urban development.

Following consultation with the UK Department for International Development (DFID) (now the UK Foreign, Commonwealth and Development Office (FCDO)) topics were agreed for three evidence and gap maps: disability (published as Saran et al. (2020b)), transport (protocol published as Malhotra et al. (2021)), and access to justice (ongoing). For the disability map, CEDIL worked with the International Centre of Evidence for Disability (ICED) at the London School of Hygiene and Tropical Medicine.

The disability map found that evidence of the effectiveness of disability-related programmes is quite scarce, and what evidence there is is geographically concentrated, coming mainly from Iran, India and Turkey. There are few studies from sub-Saharan Africa. In addition, the literature adopts a medical, rather than social, model: 'the interventions mostly try to change characteristics of the person with a disability (e.g., improve social skills) rather than to address structures (e.g., readiness of schools to include people with learning disabilities)' (Saran et al., 2020b: 3).

Disability was of particular interest to DFID as it was hosting an international summit on disability, to take place in July 2018. It was also launching two disability-related funding windows, one for implementing disability-inclusive development projects, and one for related research. Given these interests, DFID commissioned Campbell and ICED to undertake three rapid evidence assessments (REAs) based on the studies in the map, covering education, livelihoods and social inclusion (published as Kuper et al. (2018a), Kuper et al. (2018b) and White et al. (2018), respectively).

The NGO Sightsavers is implementing DFID's Disability-Inclusive Development programme. They used the information from the map regarding where there were evidence gaps, and the information from the REAs to shape their own interventions and the evaluations of those interventions. ICED, who co-authored the evidence and gap map and led on the REAs, won the DFID-awarded research contract to support the production of evidence for disability for development, which they have named the Programme for Evidence to Inform Disability Action (PENDA). Under PENDA, CEDIL updated the disability map, and is using the studies in the updated map to produce full systematic reviews from the REAs.

In the meantime, ICED was commissioned by CEDIL to produce an evidence portal, called the disability evidence portal (Figure 5), under CEDIL's 'Use of Evidence' programme of work (see Shakespeare (2020) for further details). This evidence portal is based on evidence from systematic reviews, which will have content based on the PANDE-supported reviews based on the map. CEDIL funding allowed ICED to produce a prototype of the evidence portal with eight summaries. They subsequently obtained funding from another source to take the portal to scale, producing 100 evidence briefs in three years. These briefs are prepared by ICED's partners in developing countries, thus supporting the development of disability research centres in the global south.

The development of the evidence portal involved a high level of stakeholder engagement with disabled people's organisations and FCDO staff, which will help ensure its use. 
CEDIL methods working paper: The strategic use of evidence and gap maps to build evidence architecture

\subsection{Case Studies Nos. 3 and 4: The UK What Works Centres - CHI and YEF}

$\mathrm{CHI}$ is a What Works Centre promoting the use of evidence for approaches to improving the welfare of those experiencing, or at risk of, homelessness. Evidence portals are central to the What Works model of supporting the use of research evidence by decision-makers. $\mathrm{CHI}$ approached the Campbell Secretariat to assist in generating evidence maps to support the development of their evidence portal, the Intervention Tool.

Specifically, to support the evidence portal, $\mathrm{CHI}$ commissioned a report on the evidence standards behind evidence portals (Gough and White, 2018), and two evidence maps: the effectiveness map and the implementation issues map. The first map includes studies of the effectiveness of interventions to improve the welfare of people experiencing, or at risk of, homelessness (White et al., 2020c). The current version of the effectiveness map includes just under 400 studies, organised by intervention and outcome. The implementation issues map is a map of process evaluations (White et al., 2018). The row headings of the map are interventions, using the same categories and sub-categories as are used in the effectiveness map. The column headings are not outcomes but barriers and facilitators. The current version of the map contains 275 studies.

The studies identified in the map were used to prepare the content for the first edition of the evidence portal. However, the map also showed that homelessness is an 'under-reviewed' area: of the 264 studies in the first map, only 21 - fewer than 10\% - were reviews, and most these reviews were in the health sector. The map was used to identify three priority topic areas for which reviews were commissioned from Queen's University Belfast: discharge, access to health, and accommodation-based approaches (Hanratty et al., 2020; Miller et al., 2020; and Keenan et al., 2020, respectively). The reviews were based on the studies in the map so no additional search was required.

The original UK What Works Centre, NICE, is producing a new guidance document, titled 'Integrated health and social care for people experiencing homelessness', in partnership with the Centre for Homelessness Impact. The map, and the reviews produced on the basis of the map, are being used by NICE to inform this guidance. This is the first time NICE has used an evidence and gap map. They have indicated to Campbell that they hope to do so again as a way to accelerate the process of developing guidelines, as well as building on the work of What Works Centres in related policy areas.

A similar approach has been adopted with YEF. YEF is a What Works Centre the purpose of which is preventing violence among children aged 10-14, which includes preventive approaches which can take place for children aged under 10. A team from the Campbell Collaboration Secretariat and Campbell South Asia produced an evidence and gap map (White et al., 2021a) to map these interventions. This is a very large map with 2,017 studies, of which 268 are systematic reviews. These reviews are reasonably well spread across the interventions in the map, though not all interventions are covered.

On the basis of the systematic reviews contained in the map, the Campbell team wrote 15 evidence summaries on topics such as anti-bullying interventions and sports programmes. 
CEDIL methods working paper: The strategic use of evidence and gap maps to build evidence architecture

These summaries are relatively short documents of four to six pages that summarise the findings of a specific review, or small set of reviews, on a specific topic, such as mentoring or sports interventions. These summaries have mainly been used to inform YEF's commissioning of new interventions, along with evaluations of those interventions.

Another team from Campbell and the University of Cambridge prepared 14 technical reports, again based on systematic reviews in the map, and also process evaluations from the UK and Ireland. These technical reports are longer than the evidence summaries, usually around 20 pages. Like the evidence summaries, the technical reports are primarily based on one review, which has been selected as the most relevant to the desired scope to inform an approach in YEF's toolkit, e.g. bullying. As described above in Section 3, a toolkit has three levels. These technical reports make up the third level of the toolkit and inform the content of the first two levels. Specifically, the reports give the impact estimate and evidence rating that will be used for the top level, and a list of implementation issues which are used for the second level.

The preparation of the evidence summaries and technical reports, and examination of the map itself, helped to identify topics for new systematic reviews. Three are currently being prepared, with a plan to commission a further 10-12 over the next year or so. These reviews will inform later releases of the YEF evidence portal, with additional approaches added corresponding to new technical reports based on the new reviews.

\subsection{Case Study No. 5: Child protection in institutional settings}

The foundation Porticus commissioned Giving Evidence to produce a map of interventions that address institutional child maltreatment (Finch et al., 2021). The map contains 84 papers, of which 11 are systematic reviews (plus one review update) and 62 primary studies (including three protocols). Porticus' approach to using the map is described in more detail in Fiennes et al. $(2021)^{5}$

Campbell maps usually map the evidence, without saying what the evidence says, but because Porticus wanted the work to inform its own giving in this area, as well as that of other funders (and indeed practitioners), it wanted to know what the evidence said. Therefore, Giving Evidence (supported by Campbell) also produced a 'Guidebook', which does this. The Guidebook comprises three sections:

1) a 'macro' section which discusses the evidence overall, for example the patterns around the sample size of the studies, effect estimates, the number of branded programmes studied, the paucity of information about programme cost, the risks of bias most common across the set of studies, etc.;

2) syntheses within cells which have three or more studies; and

3) summaries of individual studies for cells with only one or two studies. These summaries are short narrative reviews, which was feasible since most cells had few studies. For

\footnotetext{
${ }^{5}$ There is also a video presentation that forms part of the Campbell webinar series, which can be found on the Campbell YouTube Channel.
} 
CEDIL methods working paper: The strategic use of evidence and gap maps to build evidence architecture

example, the cell for prevention interventions (the most heavily populated row) and child social and emotional learning outcomes has just five primary studies.

Unusually, the online interactive map includes the following syntheses and summaries:

- For a cell with three or more studies, if a user clicks that cell, they get the list of studies as usual, plus an entry at the top which is the synthesis for that cell (Figure 6). A summary of that synthesis appears in the pop-up window, and there is a link to the full synthesis at the foot of that page.

- For a cell with one or two studies, if a user clicks that cell, they get the list of studies as usual. The pop-up window displays the abstract of that paper, and at the bottom is a link to the Guidebook's summary of that study.

\section{Figure 6: Including cell-wise evidence summaries in an evidence and gap map}

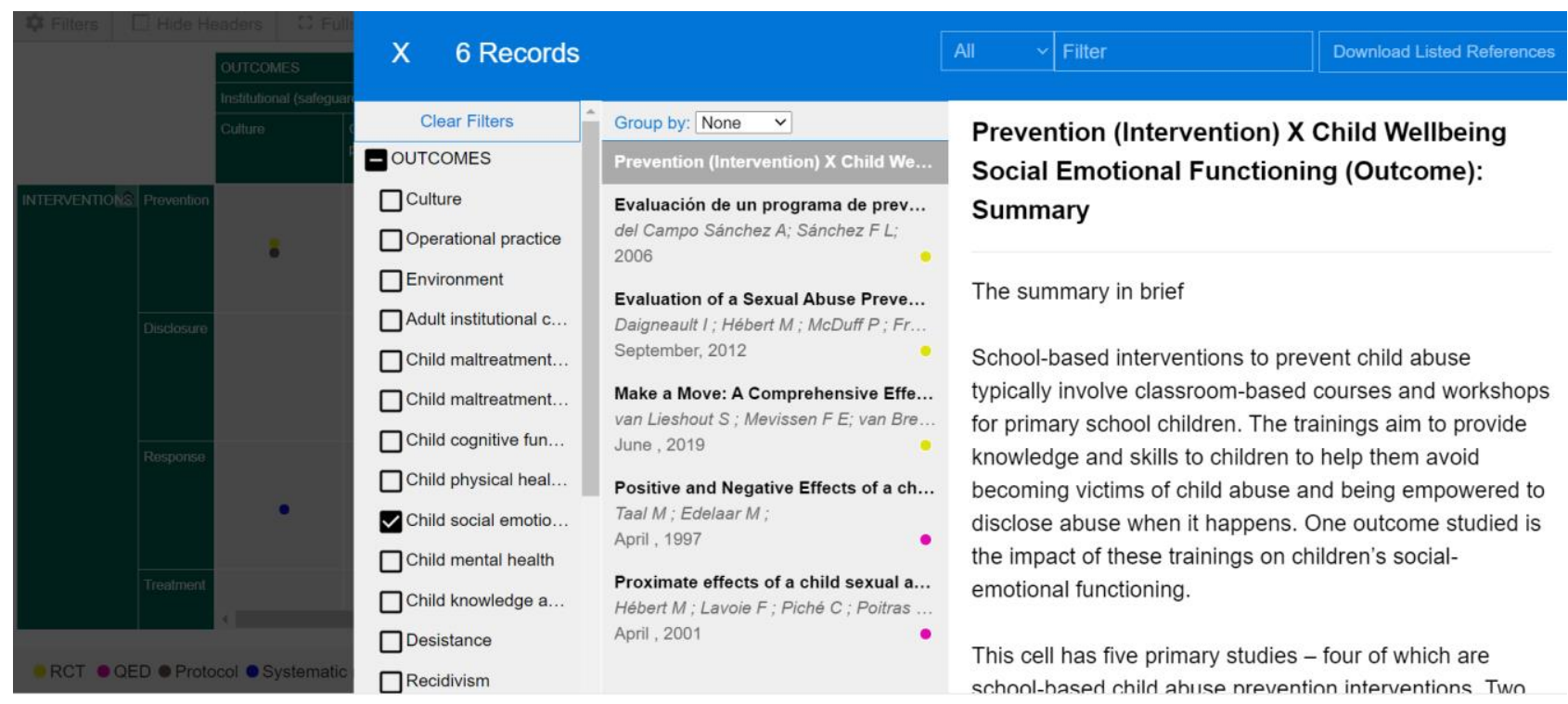

Source:

https://eppi.ioe.ac.uk/CMS/Portals/35/Nov\%2025\%20Map\%20by\%20Study\%20Design.html

In addition, Porticus and Giving Evidence analysed Porticus' grants/grantees in child protection, to assess which ones (and how many, and how much of Porticus' spend by value) are in areas which have evidence already, versus those which are in areas still without evidence. This found that:

- one set of grants is in areas which have some evidence (mainly education-related interventions);

- one set of grants is in areas without much evidence (mainly safeguarding work); and

- (a) even when there is evidence, we can have only low confidence in the findings; and (b) most of the studies are from high-income countries, whereas Porticus works mainly in middle- and low-income countries, for which there is little or no evidence.

Finally, alongside creating and using the evidence and gap map to learn from the research evidence, Porticus wanted to learn from its everyday experience. Its staff identified a set of 10 
CEDIL methods working paper: The strategic use of evidence and gap maps to build evidence architecture

priority 'learning questions' about what they need to learn to effectively implement their global strategy. Staff recorded and shared their learning in response to these questions.

Through these approaches, the map and Guidebook have supported the design of new programmes and identified evidence gaps to be filled through new evaluation studies. Porticus has already commissioned an evaluation in African countries of a wellevidenced programme for which all the evidence currently comes from the United States. 


\section{Lessons about the use of evidence and gap maps}

Evidence and gap maps have proved to be a very useful evidence synthesis product, with a range of different users. However, the key message of this paper is that producing an evidence and gap map is not an end in itself. One of the drawbacks of maps is that they show what evidence is there, but not what it says. Using the maps for knowledge brokering of the systematic reviews included in the map is a systematic approach to producing evidence-based decision-making products. Thus, in the approach advocated in this paper, an evidence map is used to survey the base of the knowledge brokering pyramid (Figure 2). Existing reviews suitable for producing evidence-based decision-making products are identified. Where reviews are not available, new reviews are commissioned based on the studies in the map. It is suggested that supplementary searches then be undertaken, with any new studies added to the map. The map will show where a review would be empty, so these studies can be added to the map.

All the examples given in this paper were commissioned by user commissioners. That is, the commissioner had planned uses for the map prior to commissioning it, and was usually involved in defining the scope and framework for the map. This involvement of the commissioner in the map production process facilitates use of the map.

Evidence and gap maps greatly increase the discoverability of evidence, being valuable collections of resources. The $\mathrm{CHI}$ homelessness maps are the world's largest online collection of homelessness evaluations, and there are many more such studies than the commissioner expected to find. In the case of the YEF map, the team expected to locate 400-600 studies, but there are over 2,000 in the first edition, with 400-500 more studies to be added. Country evaluation maps make available hundreds of evaluations, many of which have likely had a very narrow readership prior to inclusion in the map.

There are a range of evidence-based decision-making products which might be produced. The simplest are topic-specific evidence summaries based on reviews or sets of reviews in the map. These summaries may be added to the online interactive map. Such summaries may be compiled into a guidebook, as was done by Giving Evidence for Porticus. Or the map can be used as the basis for the creation of the evidence portal.

Maps are a proven way of getting evidence into use but researchers must never fall into the trap of thinking that supplying a research product alone is sufficient to ensure its use. User engagement in production of the map helps, but once the final evidence-based product is available, communication and other forms of stakeholder engagement are usually required to support uptake. In that way we can increase the number of decisions that are made based on evidence rather than preconceptions, biases and bureaucratic inertia.

This is an approach which can be - and should be - used across all sectors in all countries. Evidence and gap maps are a global public good. Sector-specific global maps should be supported by the relevant multilateral agencies, and country evaluation maps produced for every developing country, and for every state in larger countries, such as India. These maps need to be updated annually. As just stated, the supply of these maps needs to be accompanied by work on the demand side to identify the appropriate subject and type of 
CEDIL methods working paper: The strategic use of evidence and gap maps to build evidence architecture

evidence products that are then developed on the back of the maps. In this way, evidencebased decision-making will become a reality across all sectors in all countries, and we will be able to end the spending billions of dollars - and consequent lost development potential and wasted lives - on ineffective policies and programmes.

\section{Acknowledgements}

Thanks to Anjini Mishra and Jeannie Annan (IRC), Caroline Fiennes (Giving Evidence), Ligia Teixeira (CHI) and Tom Shakespeare (ICED) for additional information on their respective use of maps.

\section{References}

Fiennes, C., Krepels, J. and de Bruin Cardoso (2021) Mapping the evidence to improve grantmaking. Alliance Magazine March, pp. 23-25.

Finch, M., Featherston, R., Chakraborty, S., Bjørndal, L., Mildon, R., Albers, B., Fiennes, C., Taylor, D. J. A., Schachtman, R., Yang, T., and Shlonsky, A. (2021) Interventions that address institutional child maltreatment: evidence and gap map. Campbell Systematic Reviews 17(1), p. e1139. https://doi.org/10.1002/cl2.1139.

Gawande, A. (2009) The Checklist Manifesto, Metropolitan Books

Gough, D. and White, H. (2018) Evidence standards and evidence claims in web based research portals. London: Centre for Homelessness Impact. ISBN: 978-1-9995928-3-7

Hanratty, J, Miller, S, Keenan, C, Cowman, J, Hamilton, J, Mackie, P. (2020) PROTOCOL: Discharge programmes for individuals experiencing, or at risk of experiencing, homelessness: A systematic review. Campbell Systematic Reviews 16, p. e1109. https://doi.org/10.1002/cl2.1109.

James, K.L., Randall, N.P. and Haddaway, N.R. (2016) A methodology for systematic mapping in environmental sciences. Environ Evid 5, 7. https://doi.org/10.1186/s13750-016-0059-6.

Keenan, C., Miller, S., Hanratty, J. et al. (2020) PROTOCOL: Accommodation-based interventions for individuals experiencing, or at risk of experiencing, homelessness. Campbell Systematic Reviews 16, p. e1103. https://doi.org/10.1002/cl2.1103.

Kuper, H., Saran, A., White, H. (2018a) Rapid Evidence Assessment (REA) of What Works to Improve Educational Outcomes for People with Disabilities in Low- and Middle-Income Countries. London: ICED; and New Delhi: Campbell Collaboration.

Kuper, H., Saran, A., White, H. (2018b) Rapid Evidence Assessment (REA) of What Works to Improve Livelihood Outcomes for People with Disabilities in Low- and Middle-Income Countries. London: ICED and PENDA; and New Delhi: Campbell Collaboration.

Lum, C., Koper, C.S. and Telep, C.W. (2011) The evidence-based policing matrix.J Exp Criminol 7, pp. 3-26. https://doi.org/10.1007/s11292-010-9108-2 
CEDIL methods working paper: The strategic use of evidence and gap maps to build evidence architecture

Malhotra, S., White, H., de la Cruz, N. et al. (2021) PROTOCOL: Evidence and gap map: studies of the effectiveness of transport sector interventions in low- and middle-income countries. Campbell Systematic Reviews 17, p. e1136. https://doi.org/10.1002/cl2.1136

Miller, S., Keenan, C., Hanratty, J. et al. (2020) PROTOCOL: Improving access to health and social services for individuals experiencing, or at risk of experiencing, homelessness. Campbell Systematic Reviews 16, p. e1118. https://doi.org/10.1002/cl2.1118.

Phillips, D., Coffey, C., Tsoli, S., Stevenson, J., Waddington, H., Eyers, J., White, H. and Snilstveit, B. (2017) A map of evidence maps relating to sustainable development in low- and middleincome countries: Evidence gap map report. CEDIL Pre-Inception Paper. London: CEDIL.

Pundir, P., Saran, A., White, H., Subrahmanian, R., Adona, J. (2020) Interventions for reducing violence against children in low- and middle-income countries: An evidence and gap map. Campbell Systematic Reviews 16, p. e1120. https://doi.org/10.1002/cl2.1120

Saran, A. and White, H. (2018) Evidence and gap maps: a comparison of different approaches. Campbell Systematic Reviews 14, pp. 1-38. https://doi.org/10.4073/cmdp.2018.2

Saran, A., White, H., Albright, K., Adona, J. (2020a) Mega map of systematic reviews and evidence and gap maps on the interventions to improve child well-being in low- and middleincome countries. Campbell Systematic Reviews 16, p. e1116. https://doi.org/10.1002/cl2.1116

Saran, A., White, H., Kuper, H. (2020b) Evidence and gap map of studies assessing the effectiveness of interventions for people with disabilities in low-and middle-income countries. Campbell Systematic Reviews 16, p. e1070. https://doi.org/10.1002/cl2.1070

Shakespeare, T. (2020) Taking disability evidence to policy makers who need it. CEDIL Blog. https://cedilprogramme.org/blog/disability-evidence/

Snilstveit, B., Vojtkova, M., Bhavsar, A., Gaarder, M. (2013). Evidence gap maps: a tool for promoting evidence-informed policy and prioritizing future research. Policy Research Working Paper No. 6725. Washington, D.C.: World Bank.

https://openknowledge.worldbank.org/handle/10986/16941 License: CC BY 3.0 IGO

Sparling, T.M., White, H., Kadiyala, S. (2019) PROTOCOL: Evidence and gap map protocol: Understanding pathways between agriculture and nutrition: An evidence and gap map of tools, metrics and methods developed and applied in the last 10 years. Campbell Systematic Reviews 15, p. e1035. https://doi.org/10.1002/cl2.1035

Sparling, T.M., White, H., Boakye, S., John, D. and Kadiyala, S. (2021) Understanding pathways between agriculture and nutrition: An evidence and gap map of tools, metrics and methods developed and applied in the last 10 years. Advances in Nutrition 12(2).

White, H. (2019) The twenty-first century experimenting society: the four waves of the evidence revolution. Palgrave Commun 5, 47. https://doi.org/10.1057/s41599-019-0253-6 
CEDIL methods working paper: The strategic use of evidence and gap maps to build evidence architecture

White, H. (2020a) The global evidence architecture in health and education: a comparative scorecard. In Gorard, S. Getting Evidence into Education. London: Routledge.

White, H., Albers, B., Gaarder, M. et al. (2020b) Guidance for producing a Campbell evidence and gap map. Campbell Systematic Reviews 16, p. e1125. https://doi.org/10.1002/cl2.1125

White, H., Saran, A., Verma, A, Oprea, E. and Babudu, P. (2021a) Evidence and gap map of interventions to prevent children getting involved in violence: technical report on the first edition. London: Youth Endowment Foundation.

White, H., Lubanga, T., Rathinam F. et al. (2021b) Development evaluations in Uganda 200018: A country evaluation map. CEDIL Working Paper [in development].

White, H. Saran, A., Polack, S., and Kuper, H. (2018) Rapid evidence assessment of 'what works' to improve social inclusion and empowerment for people with disabilities in low- and middleincome countries. London: ICED, London School of Hygiene and Tropical Medicine; New Delhi: Campbell Collaboration.

White, H. Saran, A., Verma, A. and Verma, K. (2020c) The effectiveness of interventions to improve the welfare of those experiencing and at risk of homelessness: An updated evidence and gap map. Global Evidence and Gap Map of Effectiveness. Third Edition. London: Centre for Homelessness Impact.

White, H., Wood, J. and Fitzpatrick, S. (2018) Evidence and gap maps on homelessness. A launch pad for strategic evidence production and use: Part 2: Global Evidence and Gap Map of Implementation Issues. London: Centre for Homelessness Impact.

WHO (2010) WHO Handbook for Guideline Development. WHO. https://www.who.int/hiv/topics/mtct/grc handbook mar2010 1.pdf 
CEDIL methods working paper: The strategic use of evidence and gap maps to build evidence architecture

\section{Contact us}

Centre of Excellence for Development Impact and Learning (CEDIL) London International Development Centre

36 Gordon Square

WC1H OPD

www.cedilprogramme.org 
Caill:=:

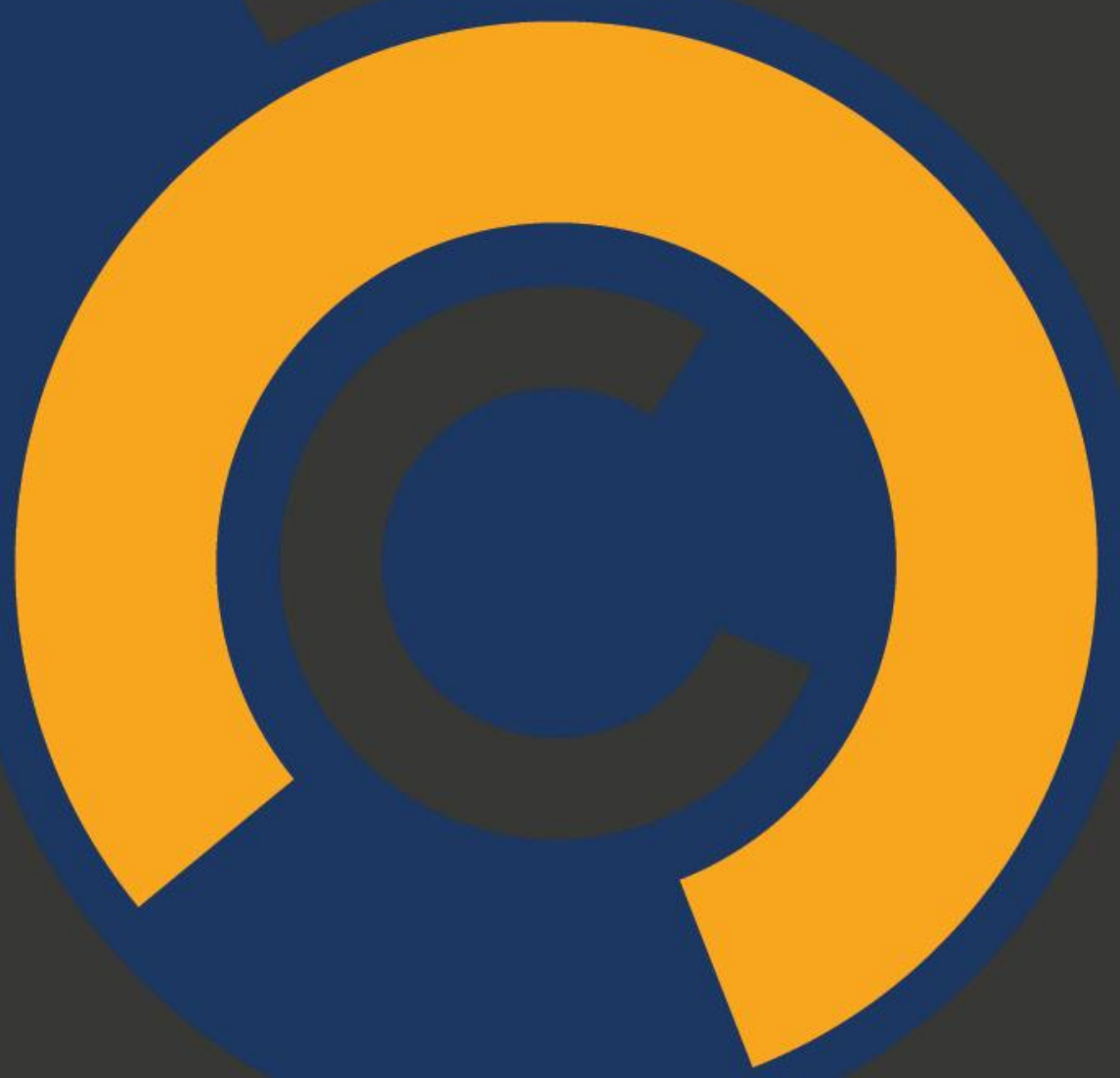

www.cedilprogramme.org 